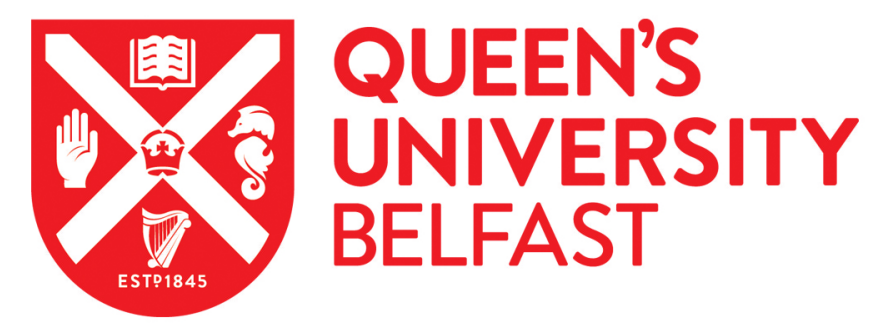

\title{
Thermal Modelling and Analysis of a Cube Satellite, EIRSAT-1: Steady Analysis
}

Wallace, P., Kalapura, A., \& Kim, S. (2018). Thermal Modelling and Analysis of a Cube Satellite, EIRSAT-1: Steady Analysis. In 2018 AIAA SPACE and Astronautics Forum and Exposition [AIAA 2018-5285] American Institute of Aeronautics and Astronautics Inc. (AIAA). https://doi.org/10.2514/6.2018-5285

Published in:

2018 AIAA SPACE and Astronautics Forum and Exposition

Document Version:

Peer reviewed version

Queen's University Belfast - Research Portal:

Link to publication record in Queen's University Belfast Research Portal

Publisher rights

Copyright 2018 AIAA This work is made available online in accordance with the publisher's policies. Please refer to any applicable terms of use of the publisher.

\section{General rights}

Copyright for the publications made accessible via the Queen's University Belfast Research Portal is retained by the author(s) and / or other copyright owners and it is a condition of accessing these publications that users recognise and abide by the legal requirements associated with these rights.

Take down policy

The Research Portal is Queen's institutional repository that provides access to Queen's research output. Every effort has been made to ensure that content in the Research Portal does not infringe any person's rights, or applicable UK laws. If you discover content in the Research Portal that you believe breaches copyright or violates any law, please contact openaccess@qub.ac.uk. 


\title{
Thermal Modelling and Analysis of a Cube Satellite, EIRSAT-1: Steady Analysis
}

\author{
Peter Wallace ${ }^{1}$ \\ Stanford University, Stanford, California, 94305, USA \\ Aby Kalapura ${ }^{2}$ \\ University College Dublin, Dublin, Ireland \\ Sung in $\mathrm{Kim}^{3}$ \\ Queen's University Belfast, Belfast, BT7 1NN, UK
}

\begin{abstract}
The Educational Irish Research Satellite, EIRSAT-1, was being developed through a collaborative space project by students and staff of University College Dublin and Queen's University Belfast with the aims to build, launch and operate the first Irish satellite. This project was selected by the European Space Agency (ESA) as a part of its educational 'Fly Your Satellite' program and it will have a significant impact on the future space industry in Ireland by giving students first-hand experience in space flight for the first time. In this paper, the results from the first stage of thermal modeling and steady analysis of EIRSAT-1, produced using the commercial software package, Thermal Desktop, are presented. An orbital analysis was performed in which three different satellite orientations were tested for a given orbit. The orbital positions for both the maximum and minimum average absorbed heat fluxes were found in which the end cap is always pointing towards the center of the Earth. An internal thermal analysis was carried out, firstly on individual components, which were then combined to give a complete system-level thermal analysis. The overall steady-state thermal analysis of the combined internal and external structure was carried out at the worst hot and cold orbital positions. As a result, the most critical component in thermal control is found. A baseline case with no thermal control was compared against thermally controlled cases - using solar paints and insulation (MLI). The cases with MLI, plus SolarWhite paint show a significant reduction in the maximum temperature compared to the baseline case. Finally, the passive thermal control design (i.e. solar paint and MLI) is sufficient to ensure mission integrity.
\end{abstract}

\section{Introduction}

The Educational Irish Research Satellite (EIRSAT-1) was a collaborative CubeSat project of University College Dublin (UCD) and Queen's University Belfast (QUB). A CubeSat is a miniature satellite made of $10 \mathrm{~cm} \times 10 \mathrm{~cm} \times$ $10 \mathrm{~cm}$ cube and a satellite comprising a single cube is called a $1 \mathrm{U}$ CubeSat. EIRSAT-1 will be a CubeSat of size $2 \mathrm{U}$. This project has been selected by the European Space Agency (ESA) in its 'Fly Your Satellite' educational program. EIRSAT-1 will become the first ever Irish Satellite in Space. The project will provide hands-on training and education for undergraduate and graduate students at UCD and QUB in all major aspects of satellite development, under expert guidance from academic and industry mentors. EIRSAT-1 system architecture is described in Fig. 1. EIRSAT-1 has two experimental payloads of GMOD, a $\gamma$-ray detector module, and EMOD, a verification module of thermal management coatings.

EIRSAT-1 will be launched from the International Space Station (ISS) and will have similar orbital parameters, $51.6^{\circ}$ beta angle and $400 \mathrm{~km}$ altitude. Beta angle is defined as the angle between the orbital plane of the spacecraft and the vector from the Earth to the Sun. A spacecraft in Earth orbit experiences a transient thermal environment with four fluctuating thermal sources, including Solar Radiation, Earth's Albedo Radiation, Earth's Infrared Radiation and

\footnotetext{
${ }^{1}$ Graduate Student, Department of Aeronautics and Astronautics, Stanford University

${ }^{2}$ Graduate Student, School of Space, Science and Technology, University College Dublin

${ }^{3}$ Lecturer, School of Mechanical and Aerospace Engineering, Queen’s University Belfast, s.kim@qub.ac.uk
} 
heat generated from the operation of its internal components. The objective of thermal design is to investigate the thermal environment encountered during the orbit and subsequently to design a thermal control system that ensures that all the subsystems remain within their allowable temperature ranges for the duration of the mission phases. The two extreme case scenarios encountered by a satellite in low earth orbit (LEO) are the hottest case and the coldest case. In the hottest case, all three external heat sources will contribute to the thermal environment, whereas in the coldest case - when the satellite has entered the Earth's shadow - only the Earth's Infrared Radiation will contribute.

In this paper, thermal modeling and steady analysis of a CubeSat, EIRSAT-1, is presented which ensures the mission integrity of EIRSAT-1 on thermal aspects. The thermal analysis carefully focuses on the allowable temperature limits of the internal components under both the operating and non-operating conditions so that an appropriate thermal control subsystem can be proposed. That ensures these limits are not exceeded at the extremely worst cases. The satellite thermal subsystem tested here is based on passive thermal control design, exploiting black and white solar paints as well as the use of multi-layer insulation (MLI).

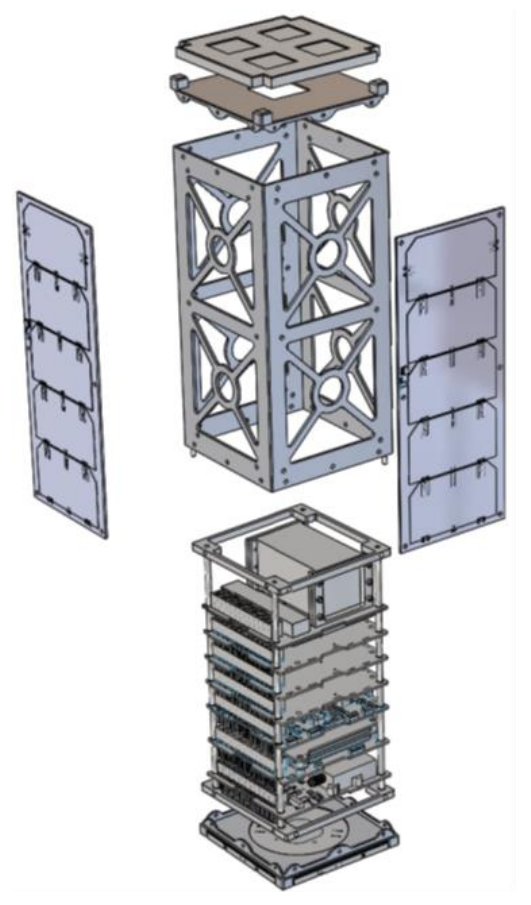

Fig. 1 EIRSAT-1 system architecture

\section{Literature Review}

A number of different groups have performed the thermal analysis of small satellites using a range of different tools. San Jose State University performed the thermal analysis of a NanoSat, where the external and internal thermal analyses were decoupled with the goal of combining both at a later stage [1]. The external orbital radiation heat flux variation during the orbits with beta angles of $30^{\circ}$ and $90^{\circ}$ and at an altitude of $400 \mathrm{~km}$ was analyzed and compared using the commercial AutoCAD software package, Thermal Desktop. The steady heat transfer analysis of internal components for the hottest case and coldest case was carried out using ANSYS Icepak. Limitations existed in this analysis, as ANSYS Icepak has difficulty in simulating the vacuum environment of space and also the spinning effect of the satellite in orbit was not considered.

Thermal analyses of a 1.5U CubeSat of EDSN (Edison Demonstration of Smallsat Networks) were performed to assess component temperatures using Thermal Desktop [2]. Initially, steady-state analysis was carried out using orbital average conditions, and then quasi-steady temperature cycles for five orbits were determined. Testing under the assumptions for many unknown radiative properties raised no significant thermal issues. Only the lower performance of the batteries was concerned due to the lower efficiency under environmental conditions that were too cold. The MHX transceiver and the Nexus phone were expected as the hottest components in the hottest case, whereas the coldest component would be the tape measure antenna in the coldest case. 
The thermal analysis of the second young engineers satellite (YES2) was conducted to assess the suitability of the thermal design of YES2 for its mission [3]. YES2 is a demonstration satellite used to evaluate the tethered space mail technology for the delivery of a small payload from LEO using a tethered re-entry capsule. The two worst cases (hot and cold steady state) as well as two transient-state analyses were conducted using ALSTOM-ThermXL software. A lumped parameter thermal network model was used considering the three axis rotations of the satellite, whereas the heat generation from the internal components was not considered. The thermal analysis verified that the satellite is safe and can withstand all thermal environments throughout the mission, in this case, eleven days.

Three different modelling methods; SolidWorks, MATLAB and Thermal Desktop, were assessed by analyzing the thermal behavior of a CubeSat, Black Knight II [4]. The SolidWorks analysis depending on a great number of assumptions showed that the temperature ranges of some components were very wide, out of the operating conditions. The MATLAB computations were more precise, and the most reasonable approach for undergraduate students unacquainted with AutoCAD. They concluded that Thermal Desktop could provide the most accurate results with considering thermal environments in space, but it required more cost, practice and time.

\section{Methodology}

The commercial AutoCAD software package, Thermal Desktop, is used for the thermal modeling and steady analysis of EIRSAT-1. Initially, the internal thermal analyses of EIRSAT-1 subsystems are separately carried out. The extreme cases are defined through the external (orbital) thermal analyses without internal subsystems. Finally, the overall CubeSat system analysis is performed using combined model of internal and external models.

\section{A. Internal Analysis}

The heat generated during the operation of the internal components within EIRSAT-1 is one of the four heat sources in orbit. As the internal components are in thermal contact with each other, conduction will be the main mechanism of thermal energy transfer. The main internal components of EIRSAT-1 are GMOD (Gamma-ray Module), EMOD (ENBIO thermal materials Module), CDH (Command and Data Handling), EPS (Electronic Power System), ADC (Attitude Determination and Control), Comms and Batteries.

The internal thermal analysis of EIRSAT-1 is first carried out at the individual component level. Finally these components are stacked in the same arrangement as in EIRSAT-1 and thermally contacted by the way of conduction through four Aluminum 6061 rods that run through the four corners of the satellite, connecting each component to the others.

The individual component analysis is carried out by modeling a simplified panel in AutoCAD/Thermal Desktop with dimensions equal to those of the component being modeled and then placing the heat source nodes at their heat generating locations. The locations of the heat-generating nodes are obtained from the dimensioned drawing of each component and the power rating and efficiency of each heat source are found on the component datasheet. An example of the thermal analysis of a simplified individual component, EPS panel, is shown in Fig. 2. The individual component analysis provides the average temperature and heat dissipation of each component, which is then thermally contacted to obtain the complete internal thermal analysis model in the system level of the CubeSat.

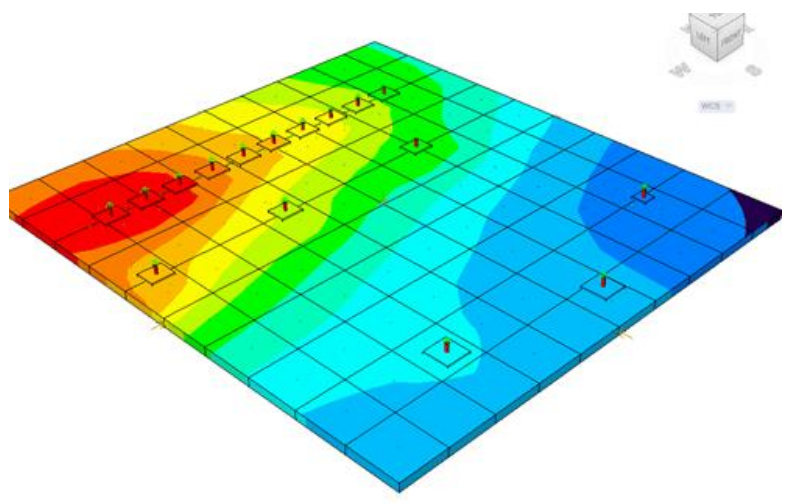

Fig. 2 Temperature distribution of an individual component analysis with the heat source nodes of the small squares with red arrows 


\section{B. Orbital Analysis}

Three orientations of an approximate cuboid model of EIRSAT-1 are tested in a circular orbit (the details of which are given in Table 1) in order to determine the extreme (hottest and coldest) cases experienced by EIRSAT-1 for use in the steady state analysis. The description of each orientation is given in Table 2.

\section{Table 1 Summary of orbital parameters}

\begin{tabular}{cc}
\hline Orbital Parameter & Value \\
\hline Altitude & $400 \mathrm{~km}$ \\
Beta Angle & 52 degrees \\
Orbital Period & $5553.62 \mathrm{~s}$ \\
Average Solar Flux & $1371 \mathrm{~W} / \mathrm{m}^{2}$ \\
Average Earth Albedo & 0.33 \\
Average Earth IR Flux & $200 \mathrm{~W} / \mathrm{m}^{2}$ \\
Space Temperature & $2.73 \mathrm{~K}$ \\
\hline
\end{tabular}

Table 2 Summary of three orientations tested

\begin{tabular}{lc}
\hline & Description \\
\hline A & End cap always pointing to the Sun \\
B & Side Panel always pointing to the Sun \\
C & End cap always pointing towards Earth's center \\
\hline
\end{tabular}

From the analysis of these three orientations cases, the worst hot and cold cases experienced by EIRSAT-1 in orbit is determined based on the orbital variation of the average heat flux absorbed by the satellite over two orbits. These hottest and coldest orbital positions are then used in the overall steady-state analysis.

\section{Optical and Thermal Properties of Key Materials}

In modeling EIRSAT-1 using Thermal Desktop, both internally and externally, the optical and thermal properties of all materials being used must be entered into the simulation.

The material of the circuit boards is fr 4oz copper, with conformal coating applied on top. The density and specific heat of fr 4oz copper are assumed as zero to make it an arithmetic node which means that it has zero capacitance and hence responds instantaneously to any change in energy balance. The initial temperature is the only requirement for this material [5]. The chip is used for the main heat dissipation element in the panel and the Aluminum 6061 is used for EIRSAT-1's internal frame structure, the end caps and the aforementioned four rods that connect the internal components to each other. MLI - Kapton is the Multi-Layer Insulation material to be used in applicable simulation cases presented in this paper.

The thermal properties of the relevant materials including thermal conductivity, specific heat and density are specified in Table 3.

The optical properties concerned within this paper are Solar Absorptivity and IR Emissivity. Optical properties are mainly a concern for those materials used externally on EIRSAT-1, as these materials are exposed to the external radiation sources mentioned previously. It is appropriate to introduce four new materials. The SolarBlack and SolarWhite paints will be used as methods of passive thermal control and the Solar Cells on the very external surface will be mounted on a PCB material. The optical properties of the relevant materials are specified in Table 4.

Table 3 Summary of thermal properties

\begin{tabular}{|c|c|c|c|}
\hline Material & Conductivity (W/m.K) & Density $\left(\mathrm{kg} / \mathrm{m}^{3}\right)$ & Heat Capacity $(\mathrm{J} / \mathrm{K})$ \\
\hline $\mathrm{Al} 6061$ & 192 & 2700 & 900 \\
\hline MLI - Kapton & 0.15 & 1420 & 1090 \\
\hline Battery & 2.49 & 3115 & 600 \\
\hline Chip & 3.60 & 2300 & 750 \\
\hline Fr 4oz Copper & 17.7 & 0 & 0 \\
\hline
\end{tabular}


Table 4 Summary of optical properties

\begin{tabular}{ccc}
\hline Material & Solar Absorptivity & IR Emissivity \\
\hline Al 6061 & 0.379 & 0.035 \\
MLI - Kapton & 0.15 & 0.05 \\
Solar Cells & 0.82 & 0.85 \\
SolarBlack & 0.95 & 0.74 \\
SolarWhite & 0.18 & 0.96 \\
PCB & 0.5 & 0.5 \\
\hline
\end{tabular}

\section{Steady Overall CubeSat Analysis}

Following the internal and orbital analyses, the internal and external structures are combined and thermally contacted through conduction between the end caps and the Aluminum 6061 rods as well as through radiation between the internal components and the inside faces of the external surface. This combined structure is then run through steady-state simulations at the worst hot and cold orbital positions - as determined by the orbital analysis - with a number of different thermal control systems being tested. This will establish the most efficient thermal subsystem design that ensures all components remain within their temperature limits.

Initially, a simplified model of the external structure of EIRSAT-1, a simple hollow cuboid of the same dimensions is used. The solar cells are represented as four individual flat panels. An example temperature contour plot showing an exploded view of this structure is shown in Fig. 3. The cases tested using this model are described briefly below.

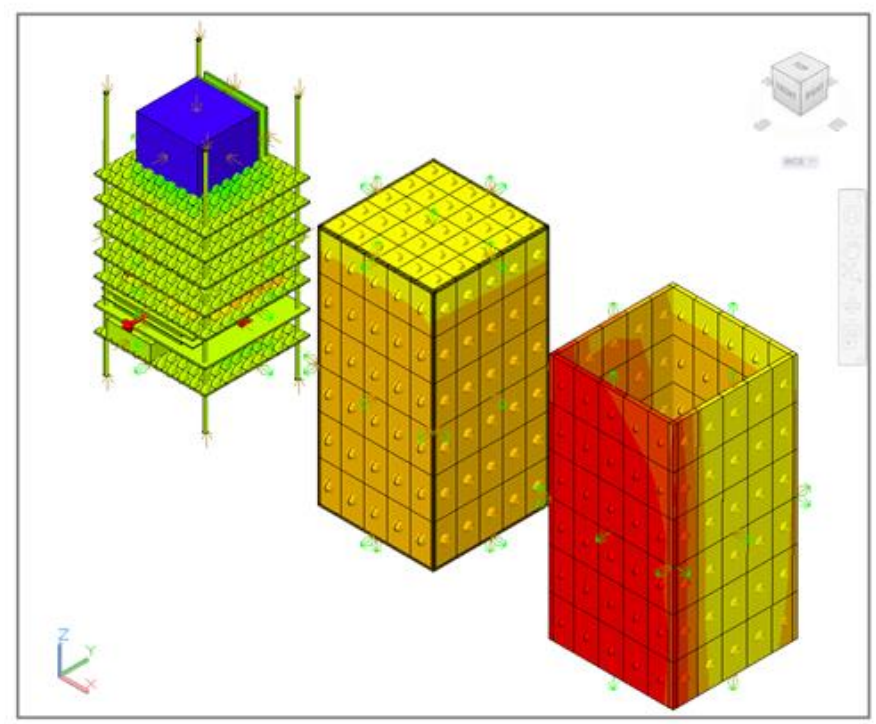

Fig. 3 Exploded view of temperature contours of the simplified EIRSAT-1 model; the internal components (left), the simple frame (middle) and the solar cells (right).

Case 1 - Baseline Case

An initial case was simulated without thermal control to act as a baseline to compare to two thermally controlled cases. This will give a good qualitative idea of the effect of adding various paints and MLI. A description of this baseline case is given below.

- $\mathrm{Al} 6061$ side panels on top of an $\mathrm{Al} 6061$ cuboid structure, which is used to model a simplified version of EIRSAT-1 frame Structure;

- $\mathrm{Al} 6061$ contactors between the end caps and $\mathrm{Al} 6061$ rods, which act as the only direct conduction paths between the internal components and the external structure;

- SolarBlack paint on the exposed $\mathrm{Al} 6061$ of the end caps. 
Case 2a - Thermally Controlled: SolarBlack Paint and MLI

- MLI side panels on top of an $\mathrm{Al} 6061$ cuboid structure, which is used to model a simplified version of the Al 6061 frame structure;

- MLI contactors between the end caps and Al 6061 rods to act as MLI between the internal components and the external structure;

- SolarBlack paint on the exposed $\mathrm{Al} 6061$ of the end caps.

Case $2 b$ - Thermally Controlled: SolarWhite Paint and MLI

- MLI side panels on top of an $\mathrm{Al} 6061$ cuboid structure, which is used to model a simplified version of the $\mathrm{Al}$ 6061 frame structure;

- MLI contactors between the end caps and Al 6061 rods to act as MLI between the internal components and the external structure;

- SolarWhite paint on the exposed $\mathrm{Al} 6061$ of the end caps.

The results of each case can be found in the Overall Analysis Results section. Following this, more simulations, again testing potential passive thermal control subsystems, will be run using a more accurate CAD model of the Aluminum 6061 frame structure in EIRSAT-1. A temperature contour plot of this more accurate frame is shown in Fig. 4.

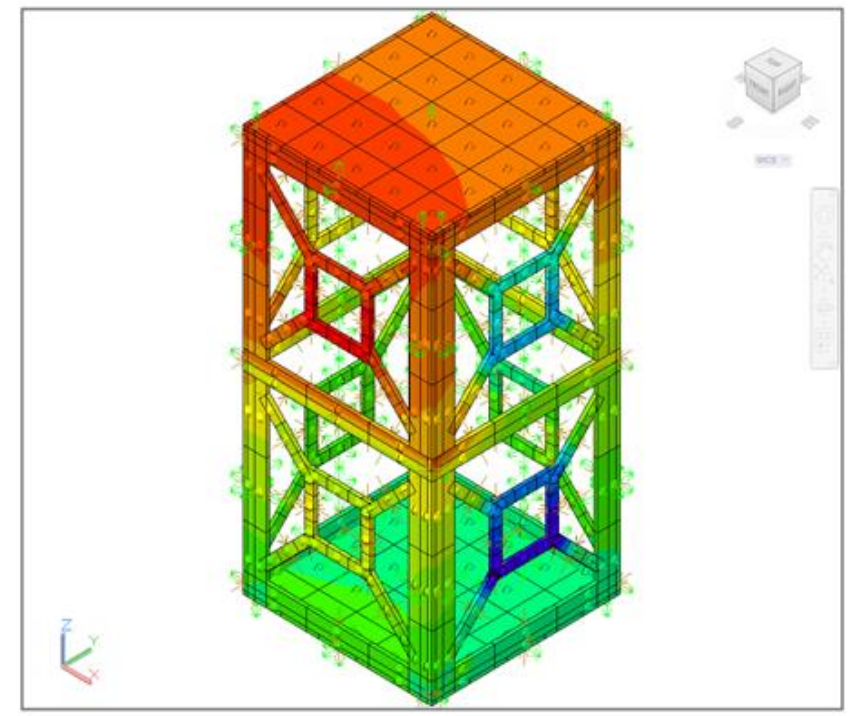

Fig. 4 Temperature contours of the more accurate Al-6061 frame model used in the final test cases

The cases that will be tested using this frame model are described briefly below.

Case 3a- SolarBlack Paint Only

- Al side panels on top of an Al 6061 modelled frame structure;

- SolarBlack paint on the exposed Al 6061.

Case $3 b$ - SolarWhite Paint Only

- $\mathrm{Al}$ side panels on top of an Al 6061 modelled frame structure;

- SolarWhite paint on the exposed Al 6061.

Case $4 \mathrm{a}$ - SolarBlack Paint and MLI

- MLI side panels on top of an Al 6061 modelled frame structure;

- MLI contactors between the end caps and Al 6061 rods to act as MLI between the internal components and the external structure;

- SolarBlack paint on the exposed Al 6061 of the end caps. 
Case 4b - SolarWhite Paint and MLI

- MLI side panels on top of an $\mathrm{Al} 6061$ modelled frame structure;

- MLI contactors between the end caps and Al 6061 rods to act as MLI between the internal components and the external structure;

- SolarWhite paint on the exposed $\mathrm{Al} 6061$ of the end caps.

The results of each case can be found in the section 'VI. Overall Analysis Results'.

\section{Orbital Analysis Results}

The peaks of the orientations A and B (described in Table 2) occur at the exactly same position in each orbit $\left(0^{\circ}\right.$ and $360^{\circ}$ ) and so for every $n$ orbits, there will be $n+1$ average heat flux peaks. The orientation $C$ forms $2 n$ peaks for every $n$ orbits because the peaks of each orbit occur at two different positions $\left(45^{\circ}\right.$ and $\left.315^{\circ}\right)$. This is shown in Fig. 5 . The peaks of the orientation $\mathrm{C}$ are shown to be symmetrical about the $0^{\circ}$ position (the south position) as expected. The projected exposure areas of EIRSAT-1's external surfaces to the Sun and Earth at the maximum and the minimum average heat fluxes for each orientation are given in Table 5. The variation of the average absorbed heat flux across all faces of EIRSAT-1 during two orbital periods for each orientation is plotted in Fig. 6.

The worst hot case for the steady-state analysis (i.e. the highest average incident heat flux) is experienced in the orientation $\mathrm{C}$ at the $45^{\circ}$ and $315^{\circ}$ orbital positions as shown by the highest peaks in Fig. 6 . The worst cold case for the steady-state analysis is experienced also in the orientation $\mathrm{C}$ at the orbital position when the satellite is in the shadow of the Earth - as shown in Fig. 6 as the sharp and sudden drop and rise in the average absorbed heat flux as EIRSAT1 passes into and out of the Earth's shadow, respectively. This can be deduced from the fact that it is in this orientation that the smallest projected satellite surface area is exposed to the Earth at min heat flux as shown in Table 5. This is the only relevant exposure area as the Earth's IR radiation is the only external heat source, whilst EIRSAT-1 is in its shadow as shown in Fig. 7. When the overall CubeSat analysis is run, these two orbital positions are to be used to find the worst hot and cold temperatures for each case.

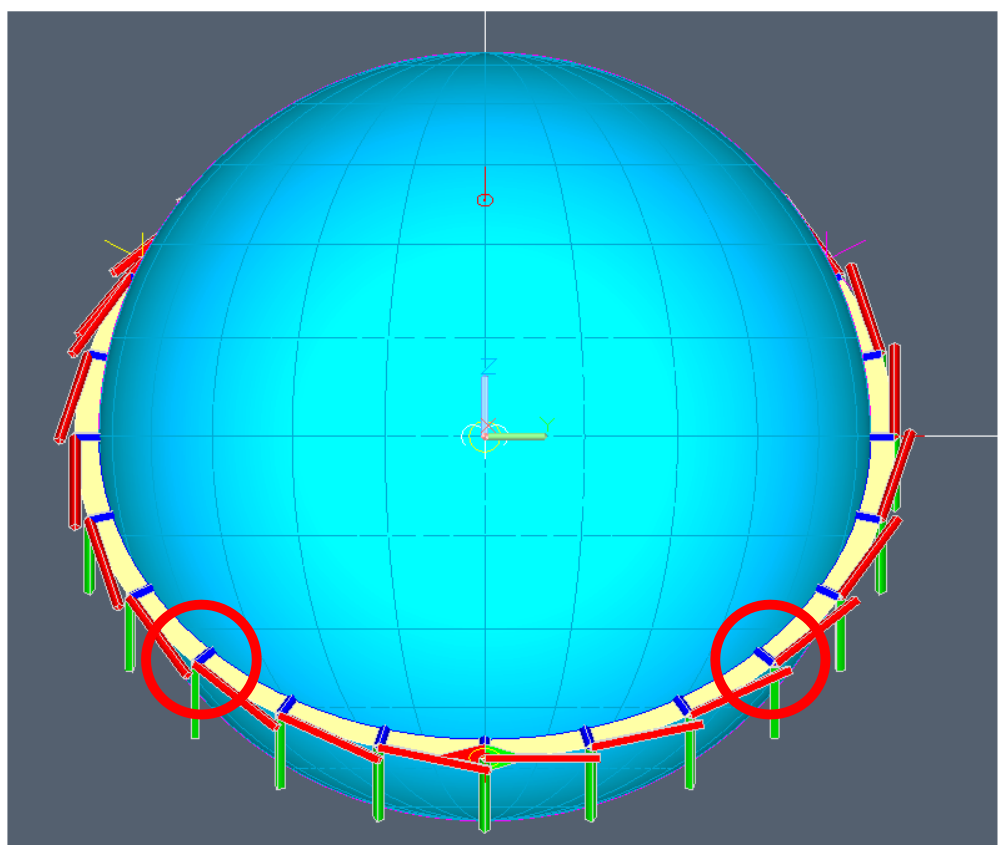

Fig. 5 EIRSAT-1 basic orbit from the point of view of the Sun in the orientation $C$ and the circled positions of the max average heat flux 
Table 5 Projected exposure areas of EIRSAT-1 for each orientation

\begin{tabular}{cccc}
\hline Orientation & Solar Exposure at Max Heat Flux $\left(\mathbf{m}^{2}\right)$ & $\begin{array}{c}\text { Earth Exposure at } \\
\text { Max Heat Flux }\left(\mathbf{m}^{2}\right)\end{array}$ & $\begin{array}{c}\text { Earth Exposure at Min } \\
\text { Heat Flux }\left(\mathbf{m}^{2}\right)\end{array}$ \\
\hline A & 0.010 & 0.022 & 0.022 \\
B & 0.020 & 0.020 & 0.020 \\
C & 0.038 & 0.010 & 0.010 \\
\hline
\end{tabular}

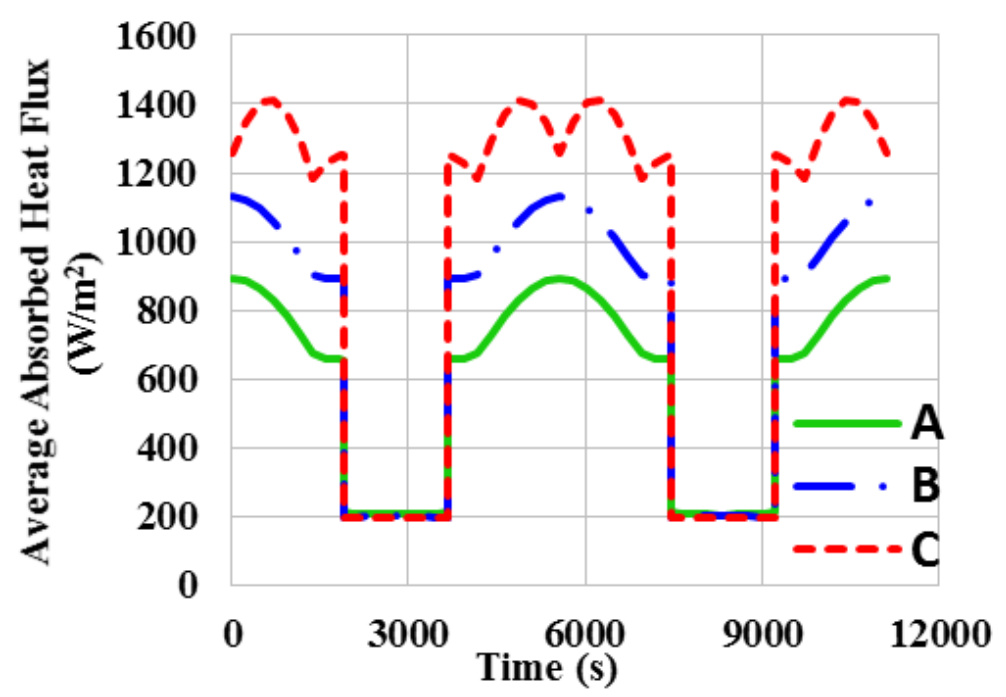

Fig. 6 Variation of average heat flux incident for three different orientations over two orbital periods

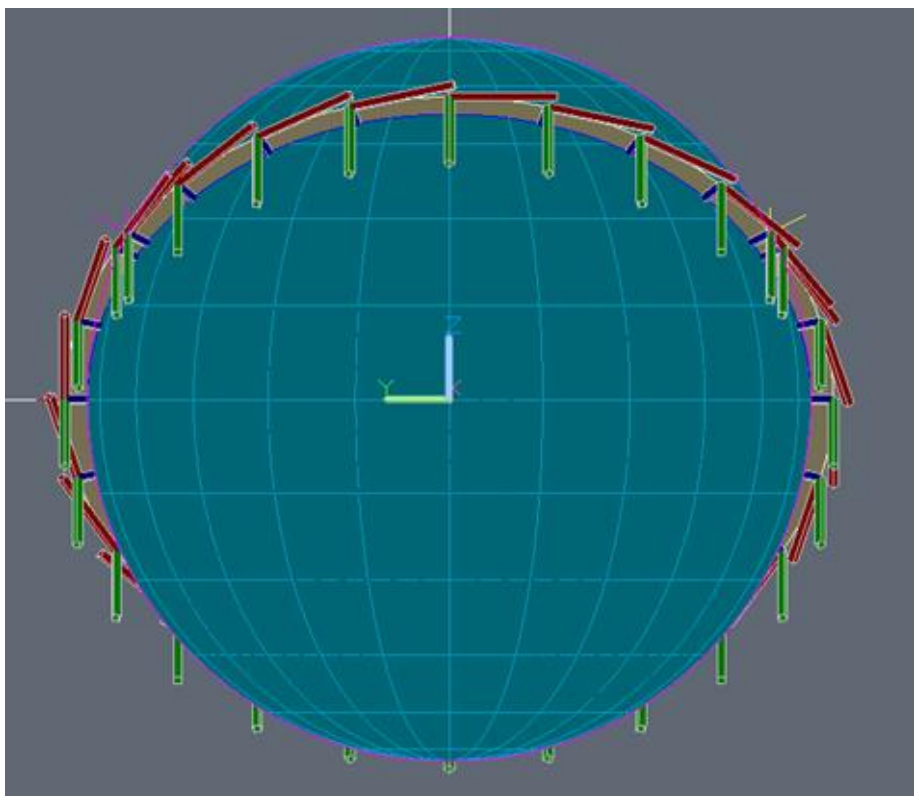

Fig. 7 EIRSAT-1 orbit in the orientation $C$ from behind the earth showing EIRSAT-1 in its shadow 


\section{Required Temperature Ranges}

\section{A. Operational Temperature Range}

The operational temperature range is the range of thermal conditions within that a component can withstand during the operation and still perform its intended task fully and accurately. The operational temperature ranges for each internal component in EIRSAT-1 is shown in Table 6.

Table 6 Operational temperature ranges of EIRSAT-1 internal components

\begin{tabular}{ccc}
\hline Component & Min $\left({ }^{\circ} \mathbf{C}\right)$ & Max $\left({ }^{\circ} \mathbf{C}\right)$ \\
\hline GMOD - SiPMs & -40 & 85 \\
GMOD - FPGA & -40 & 85 \\
EMOD - AT Mega 328 & -40 & 85 \\
EMOD - Thermocouple & -40 & 125 \\
CDH & -40 & 80 \\
ADC & -40 & 55 \\
EPS & -40 & 85 \\
Comms & -25 & 61 \\
Batteries & -10 & 50 \\
\hline
\end{tabular}

\section{B. Non-Operational Temperature Range}

The non-operational (or survival) temperature range is the range of thermal conditions within that a component can be exposed to at any time during its orbit while not in operation and still be reasonably expected to perform its intended task fully and accurately later in the mission phase. The non-operational temperature ranges for each internal component in EIRSAT-1 is shown in Table 7.

Table 7 Non-Operational temperature ranges of EIRSAT-1 internal components

\begin{tabular}{ccc}
\hline Component & Min $\left({ }^{\circ} \mathbf{C}\right)$ & Max $\left({ }^{\circ} \mathbf{C}\right)$ \\
\hline GMOD - SiPMs & $\mathrm{n} / \mathrm{a}$ & $\mathrm{n} / \mathrm{a}$ \\
GMOD - FPGA & $\mathrm{n} / \mathrm{a}$ & 105 \\
EMOD - AT Mega 328 & -40 & 105 \\
EMOD - Thermocouple & -65 & 150 \\
CDH & -40 & 85 \\
ADC & -40 & 85 \\
EPS & -50 & 100 \\
Comms & -40 & 85 \\
Batteries & -20 & 50 \\
\hline
\end{tabular}

\section{Overall Analysis Results}

The following section documents the results of the overall CubeSat analysis cases as described in the Methodology section.

\section{A. Case 1- Baseline Case}

The maximum and minimum temperatures of each component for Baseline Case are listed in Table 8 , and its temperature distribution is shown in Fig. 8. As can be seen in Table 8, two components, ADC and Batteries, show that their maximum temperatures are within the thermal margin $\left(\Delta \leq 10^{\circ}\right.$, within $10^{\circ} \mathrm{C}$ of the temperature difference from the limit temperature). The component, Batteries, is found to be the most critical component in the design of thermal control system. Eventually, appropriate and optimal thermal control system is required to satisfy the temperature limits of all the subsystems. Different configurations of passive thermal control system have been tested and discussed in the following sections to find an optimum configuration 
Table 8 Summary of the external and internal temperatures for Case 1, Baseline Case

\begin{tabular}{cccc}
\hline Component & Min $\left({ }^{\circ} \mathbf{C}\right)$ & $\operatorname{Max}\left({ }^{\circ} \mathbf{C}\right)$ & Inside Operational Range? \\
\hline External & 11.8 & 52.4 & - \\
GMOD & 7.8 & 52.3 & $\mathrm{Y}$ \\
EMOD & 11.6 & 56.8 & $\mathrm{Y}$ \\
CDH & 11.6 & 55.8 & $\mathrm{Y}$ \\
ADC & 11.6 & 54.2 & $\mathrm{Y}\left(\Delta \leq 5^{\circ}\right)$ \\
EPS & 11.6 & 61.2 & $\mathrm{Y}$ \\
Comms & 11.6 & 52.3 & $\mathrm{Y}$ \\
Batteries & 11.6 & 52.1 & $\mathrm{~N}\left(\Delta \leq 5^{\circ}\right)$ \\
Internal Ave. & 11.1 & 55.0 & -
\end{tabular}

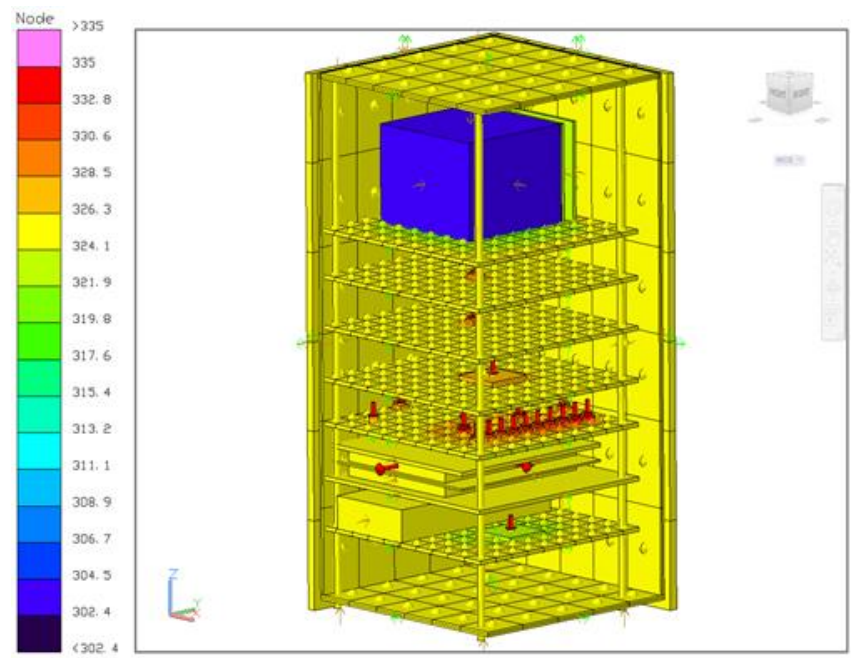

Fig. 8 Case1: Baseline - Temperature contours of EIRSAT-1 thermal model at the hottest orbital position

\section{B. Case 2a/Case2b - Thermally Controlled: SolarBlack Paint \& MLI / SolarWhite Paint \& MLI}

Table 9 gives a summary of the results obtained for Cases $2 a$ and $2 b$. As can be seen from the final column, both designs are shown to be valid as no component came within the thermal margin $\left(\Delta \leq 10^{\circ}\right.$, within $10^{\circ} \mathrm{C}$ of the temperature difference from their respective upper or lower operational temperature limits) for neither the worst hot nor worst cold cases. The additional insulation by MLI results in the significant reduction of the maximum temperatures of the components. When compared to the baseline case, the addition of MLI to the structure and SolarWhite paint, led to around $8^{\circ} \mathrm{C}$ and $12^{\circ} \mathrm{C}$ reductions in the average maximum internal temperatures, respectively.

Consequently, around $4^{\circ} \mathrm{C}$ reduction in the average maximum internal temperature is seen when using the SolarWhite paint, as opposed to the SolarBlack paint. Fig. 9 shows a comparison of the temperature contours at the worst hot case for Cases 2a and 2b. The effect of the SolarWhite paint is evident compared to the case of SolarBlack paint. The overall red colors produced by Case $2 \mathrm{a}$ (SolarBlack paint) indicates the higher temperatures as compared to the lighter (yellow and green) colors of lower temperatures in Case $2 \mathrm{~b}$ (SolarWhite paint).

Table 9 Summary of Case 2a and Case 2b results - SolarBlack versus SolarWhite

\begin{tabular}{cccccc}
\hline \multirow{2}{*}{ Case } & \multicolumn{2}{c}{ External Range $\left({ }^{\circ} \mathbf{C}\right)$} & \multicolumn{2}{c}{ Internal Range $\left({ }^{\circ} \mathbf{C}\right)$} & \multirow{2}{*}{ Valid Design? } \\
\cline { 2 - 5 } & Min & Max & Min & Max & \\
\hline 2a-Black & 11.0 & 54.2 & 11.3 & 47.3 & Y \\
2b-White & 11.2 & 51.5 & 11.4 & 43.2 & Y \\
\hline
\end{tabular}




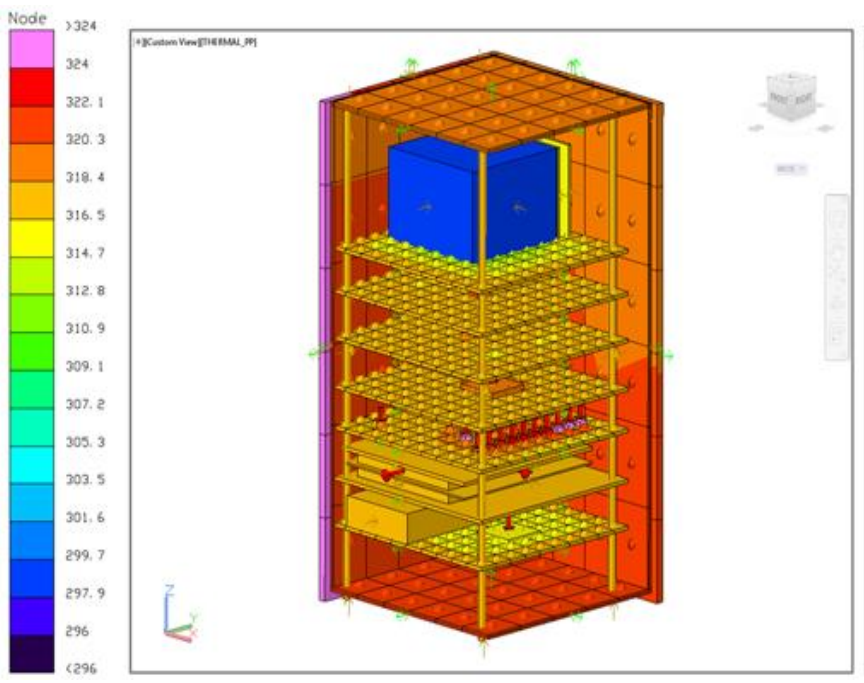

a) Case 2a (SolarBlack paint \&MLI)

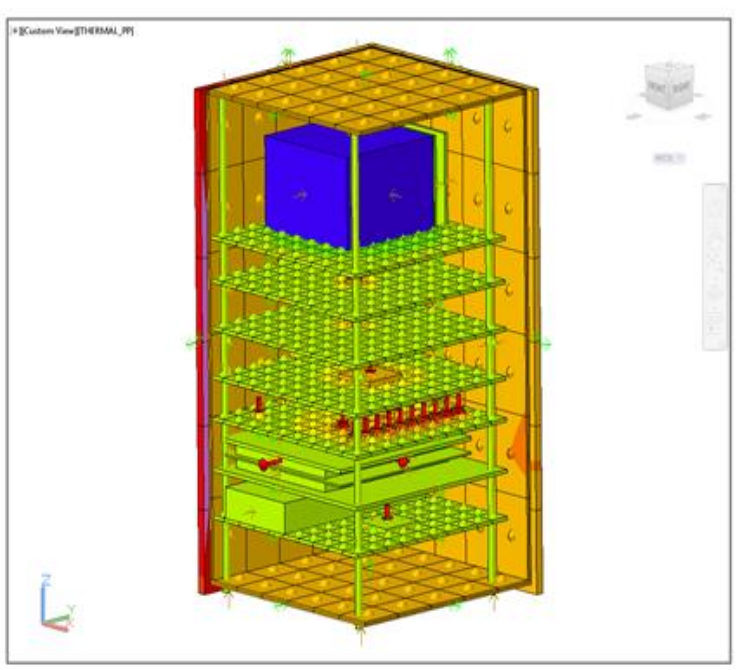

b) Case 2b (SolarWhite Paint \& MLI)

Fig. 9 Temperature contours of the EIRSAT-1 (Cuboid structure) at the hottest orbital position

C. Case 3a - More accurate Al 6061 Frame Model with SolarBlack Paint only

Table 10 Summary of the external and internal temperatures for Case 3a (SolarBlack)

\begin{tabular}{cccc}
\hline Component & Min $\left({ }^{\circ} \mathbf{C}\right)$ & Max $\left({ }^{\circ} \mathbf{C}\right)$ & Inside Operational Range? \\
\hline External & 30.35 & 65.05 & - \\
GMOD & 30.25 & 64.75 & $\mathrm{Y}$ \\
EMOD & 30.25 & 69.25 & $\mathrm{Y}$ \\
CDH & 30.25 & 68.35 & $\mathrm{Y}$ \\
ADC & 30.25 & 66.75 & $\mathrm{~N}\left(\Delta>10^{\circ}\right)$ \\
EPS & 30.25 & 73.65 & $\mathrm{Y}$ \\
Comms & 30.25 & 64.75 & $\mathrm{~N}\left(\Delta \leq 5^{\circ}\right)$ \\
Batteries & 30.25 & 64.65 & $\mathrm{~N}\left(\Delta>10^{\circ}\right)$ \\
Internal Av. & 30.25 & 67.45 & -
\end{tabular}

D. Case 3b - More accurate Al 6061 frame Model with SolarWhite Paint only

Table 11 Summary of the external and internal temperatures for Case 3b (SolarWhite)

\begin{tabular}{cccc}
\hline Component & Min $\left({ }^{\circ} \mathbf{C}\right)$ & Max $\left({ }^{\circ} \mathbf{C}\right)$ & Inside Operational Range? \\
\hline External & 30.05 & 65.05 & - \\
GMOD & 29.45 & 61.45 & $\mathrm{Y}$ \\
EMOD & 30.05 & 66.15 & $\mathrm{Y}$ \\
CDH & 30.05 & 61.65 & $\mathrm{Y}$ \\
ADC & 30.05 & 63.75 & $\mathrm{~N}\left(\Delta>5^{\circ}\right)$ \\
EPS & 30.05 & 70.55 & $\mathrm{Y}$ \\
Comms & 30.05 & 61.55 & $\mathrm{~N}\left(\Delta \leq 5^{\circ}\right)$ \\
Batteries & 30.05 & 61.45 & $\mathrm{~N}\left(\Delta>10^{\circ}\right)$ \\
Internal Ave. & 29.96 & 63.79 & - \\
\hline
\end{tabular}




\section{E. Case 3a and 3b comparison}

The maximum and minimum temperatures of each component for two cases, Case 3a (SolarBlack paint used) and Case 3b (SolarWhite paint used) are listed in Table 10 and Table 11, respectively. Fig. 10 shows a comparison of the temperature contours at the worst hot case for Case $3 \mathrm{a}$ and Case $3 \mathrm{~b}$ where different solar paints are used. Regarding the effect of different solar paints, around $4^{\circ} \mathrm{C}$ reduction in the average maximum internal temperature value is seen (in Table 10 and Table 11), when using the SolarWhite paint, as opposed to the SolarBlack paint. This reduction is also clearly seen from the color differences in the contour plots in Fig. 10. The overall darker (orange) color produced by Case 3a (SolarBlack paint) indicates the higher temperatures as compared to the lighter (yellow and green) colors of the lower temperatures in Case $3 \mathrm{~b}$ (SolarWhite paint). Similar to Case 2 (Cuboid structure), around $4^{\circ} \mathrm{C}$ reduction in the average maximum internal temperature is seen again in Case 3 (Frame structure) when using the SolarWhite paint, compared to the SolarBlack paint.

All the minimum temperatures are within the allowable temperature range. The last column in Tables 10 and 11 indicates whether the maximum temperature is within the limit. Neither design case (only solar paint used) is shown to be valid in the steady state simulations of the worst cases because in both cases the ADC, Comms system and Batteries exceed their maximum allowable operational temperatures. Similar to the Baseline Case, the component of Batteries is again found to be the most critical component with respect to thermal control system design, since its maximum temperature is the farthest beyond its temperature limit $\left(\Delta>10^{\circ} \mathrm{C}\right.$ in both cases). Therefore, additional method of thermal control is required to guarantee that all the temperatures of components including Batteries should be within their limits at the worst cases.

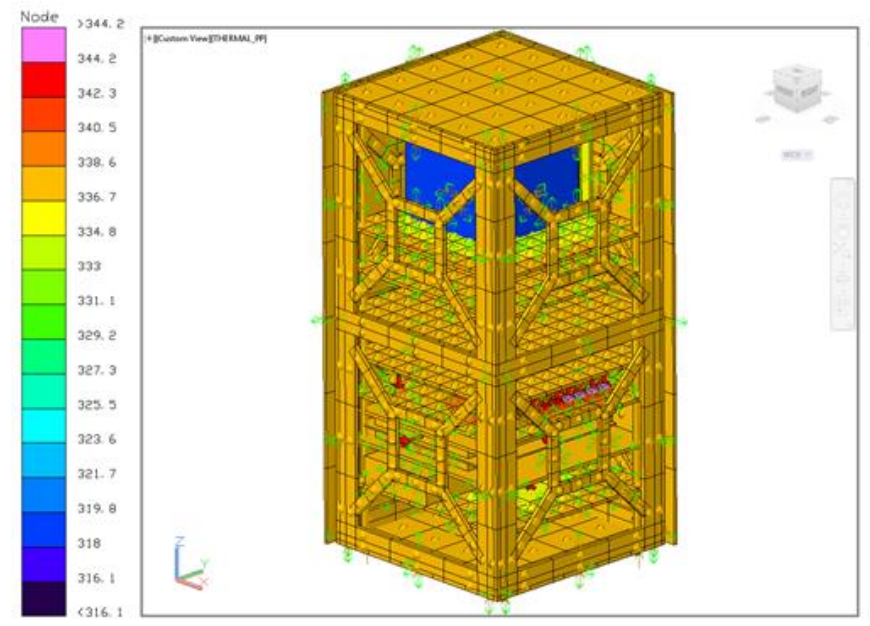

a) Case 3a (SolarBlack paint)

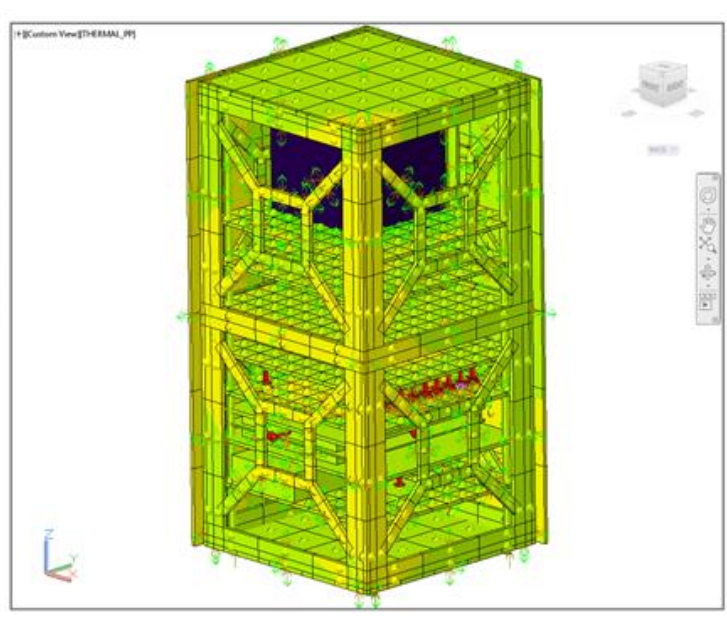

b) Case 3b (SolarWhite paint)

Fig. 10 Temperature contours of the EIRSAT-1 (Frame structure) at the hottest orbital position

F. Case 4a - More accurate Al 6061 Frame Model with SolarBlack Paint and MLI

Table 12 Summary of the external and internal temperatures for Case 4a

\begin{tabular}{cccc}
\hline Component & Min $\left({ }^{\circ} \mathbf{C}\right)$ & Max $\left({ }^{\circ} \mathbf{C}\right)$ & Inside Operational Range? \\
\hline External & 27.35 & 74.35 & - \\
GMOD & 27.25 & 50.95 & $\mathrm{Y}$ \\
EMOD & 30.25 & 55.45 & $\mathrm{Y}$ \\
CDH & 30.25 & 54.55 & $\mathrm{Y}$ \\
ADC & 30.25 & 52.95 & $\mathrm{Y}\left(\Delta \leq 5^{\circ}\right)$ \\
EPS & 30.25 & 59.45 & $\mathrm{Y}$ \\
Comms & 30.25 & 50.25 & $\mathrm{Y}$ \\
Batteries & 30.25 & 49.65 & $\mathrm{Y}\left(\Delta \leq 5^{\circ}\right)$ \\
Internal Av. & 29.82 & 53.41 & -
\end{tabular}


G. Case 4b - More accurate Al 6061 frame Model with SolarWhite Paint and MLI

Table 13 Summary of the external and internal temperatures for Case $4 \mathrm{~b}$

\begin{tabular}{cccc}
\hline Component & Min $\left({ }^{\circ} \mathbf{C}\right)$ & Max $\left({ }^{\circ} \mathbf{C}\right)$ & Inside Operational Range? \\
\hline External & 26.55 & 74.35 & - \\
GMOD & 26.85 & 39.25 & $\mathrm{Y}$ \\
EMOD & 29.75 & 44.95 & $\mathrm{Y}$ \\
CDH & 29.75 & 43.95 & $\mathrm{Y}$ \\
ADC & 29.75 & 42.35 & $\mathrm{Y}\left(\Delta>10^{\circ}\right)$ \\
EPS & 29.75 & 49.15 & $\mathrm{Y}$ \\
Comms & 29.75 & 39.25 & $\mathrm{Y}$ \\
Batteries & 29.75 & 39.25 & $\mathrm{Y}\left(\Delta>10^{\circ}\right)$ \\
Internal Ave. & 29.34 & 42.59 & - \\
\hline
\end{tabular}

H. Case 4a and 4b comparison (for different soar paints)

Tables 12 and 13 show the minimum and maximum temperatures of each component at the worst hot and cold conditions for Case 4a and Case 4b, respectively. As can be seen in Table 12, Case 4a (SolarBlack paint and MLI used) is shown to be marginally acceptable with the maximum temperatures of both critical components, ADC and Batteries, lying below the operational upper limits, but within the thermal margin $\left(\Delta<10^{\circ} \mathrm{C}\right)$. In contrast, Case $4 \mathrm{~b}$ (SolarWhite paint and MLI used) in Table 13 is shown to be comfortably acceptable since all the components including $\mathrm{ADC}$ and Batteries have a sufficient thermal margin $\left(\Delta>10^{\circ} \mathrm{C}\right)$.

A comparison of the temperature contours at the worst hot condition for Case $4 \mathrm{a}$ and Case $4 \mathrm{~b}$, where different solar paints are used, is made in Fig. 11. Fig. 11 shows clearly the color differences in the contour plots between Case $4 \mathrm{a}$ and Case $4 \mathrm{~b}$ because of the effect of different solar paints. From this comparison, a significant reduction (around $10^{\circ} \mathrm{C}$ ) in the maximum average internal temperature value is seen when using the SolarWhite paint, as opposed to the SolarBlack paint. Eventually, Case $4 \mathrm{~b}$ of passive thermal controlling by using SolarWhite paint and MLI is to be the appropriate passive thermal control system for EIRSAT-1 which ensure satisfactory thermal environment in EIRSAT1 's mission phases.

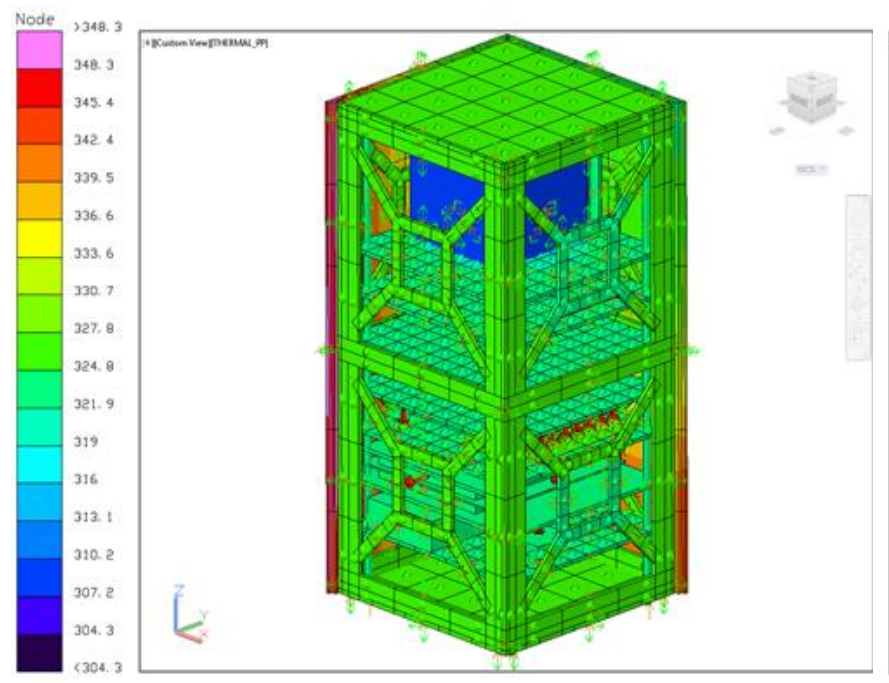

a) Case 4a (SolarBlack paint \& MLI)

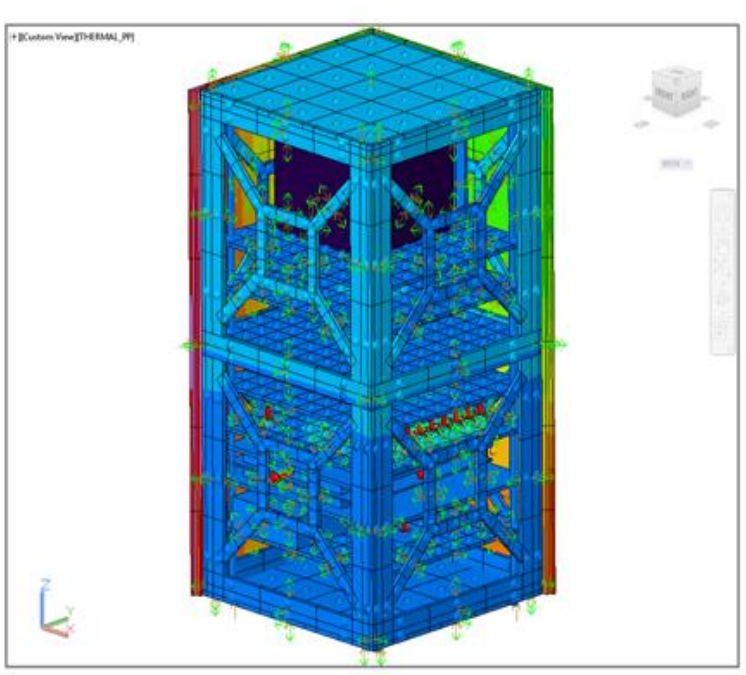

b) Case 4b (SolarWhite paint \& MLI)

Fig. 11 Temperature contours of the EIRSAT-1 (Frame structures) at the hottest orbital position 


\section{Case 3 and Case 4 comparison (effect of MLI)}

Internally higher temperature than external temperature is predicted when using the SolarBlack paint without insulation (Case 3a) because this paint absorbs more heat flux from the external radiative heat sources as compared to the SolarWhite paint. Due to the larger emissivity of the SolarWhite paint compared to the SolarBlack paint, more internal heat is allowed to escape through the external structure to the surroundings (Case 3b). Consequently, lower internal temperature than external temperature is estimated in Case 3b. In Case 3 (without insulation), the internal systems thermally interact with all the external surfaces. As a result, the internal and external temperatures of Case 3 in the steady analysis are close.

Contrary to the close temperatures between internal and external in Case 3, Case 4 shows large difference between internal and external temperatures due the insulation, MLI, as expected. A much more significant reduction in the maximum average internal temperature going from the SolarBlack to the SolarWhite paint is recorded when using MLI (Case 4a to Case 4b) as compared to without MLI (Case 3a to Case 3b). This is attributed to the insulation effect of blocking internal thermal interactions with the side walls in Case 4. The effect of different solar paints on both end caps is lessened by the thermal interactions with the side walls in Case 3. However, in Case 4, the effect of different solar paints on both end caps outstands due to thermal isolation from the side walls by the insulation, MLI.

\section{Limitations and Future Work}

Certain limitations on the accuracy of the current preliminary results exist and it is an aim to address limitations and, improve upon the results in the next stage. These limitations are outlined in this section.

The assumptions of the fixed orbital parameters lead to a number of limitations in the analysis:

- Annual variations in Solar Flux due to the eccentricity of Earth's orbit are not accounted for, but rather, the worst hot and cold cases presented are based on an annual average value. As such, higher actual hottest values are expected when Earth is closest to the Sun and, similarly, lower actual coldest values are expected when the Earth is furthest from the Sun;

- EIRSAT-1 is assumed not to spin about any of its axes in orbit. The effect of changing orientations was discussed earlier in the results section. Possible spinning of the CubeSat would create a constantly changing orientation. This should be considered in future analysis.

It is worth noting that, due to time constraints caused by the first CDR deadline of EIRSAT-1 project; every result presented in this paper is from the steady state analysis at the hottest/coldest orbital position. As a result, each temperature would be more extreme than that in reality as the hottest case does not take into account the periodic cooling effect of the Earth's shadow in orbit and similarly the coldest case does not take into account the periodic heating effect of direct solar exposure in orbit. Therefore, transient analysis with considering these periodic cooling and heating effects and seasonal variation of heat fluxes will be performed in the next stage.

\section{Conclusion}

The Educational Irish Research Satellite, EIRSAT-1, was being developed through a collaborative project by students and staff of University College Dublin (UCD) and Queen's University Belfast (QUB) with the aims to build, launch and operate the first Irish satellite. This project has been selected by the European Space Agency (ESA) as a part of its educational 'Fly Your Satellite' program. In this paper, the results from the first stage of thermal modeling and analysis of EIRSAT-1, produced using the AutoCAD commercial software package and thermal analysis tool, Thermal Desktop, were presented. Firstly, an orbital analysis was performed in which three different satellite orientations were examined for a given basic orbit. The orbital positions for both the maximum and minimum average absorbed heat fluxes were found to occur in an orbit in which the end cap is always pointing towards the center of the Earth. Finally, the overall steady-state thermal analysis of the combined internal and external structure was carried out at the worst hot and cold orbital positions. From the steady thermal analysis, the component of Batteries is found to be the most critical subsystem in thermal control system design. A baseline case of no thermal control was compared against two thermally controlled cases - using solar paints and MLI (multi-layer insulation). The cases with MLI, and plus SolarWhite paint show the reductions in the maximum temperature of Batteries; about $12^{\circ} \mathrm{C}$ and $22^{\circ} \mathrm{C}$ from that of Baseline Case, respectively. The most significant outcome of the present preliminary analysis is that passive thermal control designs (i.e. SolarWhite paint and MLI) are sufficient to ensure mission integrity 


\section{Acknowledgments}

The authors would like to sincerely thank C\&R Technologies, Inc. for the use of their software, Thermal Desktop, and their technical support during this project.

\section{References}

[1] Dinh, D. Q., “Thermal modeling of nanosat,” MSc Thesis, San Jose State University, 2012.

[2] Coker, R. F., "Thermal modeling in support of the Edison demonstration of smallsat networks project," $43^{\text {rd }}$ International Conference on Environmental System (ICES), Vail, CO, USA, 2013.

[3] Drakonakis, V., Miaris, A., Vavouliotis, A., Patricio, R., Kruijff, M., and Kostopoulos, V., "Thermal modelling of E.S.A second young engineers satellite," 57th International Astronautical Congress, Valencia, Spain, 2006.

[4] Oliver, R., Crawford, B. G., and Burrow, G. M., "Optimized undergraduate thermal analysis of cube satellites,” 13th International Energy Conversion Engineering Conference, Orlando, USA, 2015.

[5] C\&R Technologies, Inc. “Thermal Desktop User's Manual, Version 5.8”. Cullimore and Ring Technologies, Inc., June 2015 